\title{
The Effects of Implicit and Explicit Motor Learning in Gait Rehabilitation of People After Stroke: Protocol for a Randomized Controlled Trial
}

\author{
Li-Juan Jie ${ }^{1,2,3}$, MSc; Melanie Kleynen ${ }^{1,2}, \mathrm{PhD}$; Kenneth Meijer ${ }^{3}, \mathrm{PhD}$; Anna Beurskens ${ }^{2,4}, \mathrm{PhD}$; Susy Braun ${ }^{1,2}, \mathrm{PhD}$ \\ ${ }^{1}$ Research Center of Nutrition, Lifestyle and Exercise, Faculty of Health, Heerlen, Netherlands \\ ${ }^{2}$ Caphri School for Public Health and Primary care, Maastricht University, Maastricht, Netherlands \\ ${ }^{3}$ Nutrim School for Nutrition and Translational Research in Metabolism, Department of Nutrition and Movement Sciences, Maastricht University, \\ Maastricht, Netherlands \\ ${ }^{4}$ Research Centre for Autonomy and Participation of Persons with a Chronic Illness, Zuyd University of Applied Sciences, Heerlen, Netherlands
}

\section{Corresponding Author:}

Li-Juan Jie, MSc

Research Center of Nutrition, Lifestyle and Exercise

Faculty of Health

Zuyd University of Applied Sciences

Heerlen, PO Box 550

Netherlands

Phone: 31454006538

Email: 1i-juan.jie@zuyd.nl

\begin{abstract}
Background: A significant part of neurological rehabilitation focuses on facilitating the learning of motor skills. Training can adopt either (more) explicit or (more) implicit forms of motor learning. Gait is one of the most practiced motor skills within rehabilitation in people after stroke because it is an important criterion for discharge and requirement for functioning at home.

Objective: The aim of this study was to describe the design of a randomized controlled study assessing the effects of implicit motor learning compared with the explicit motor learning in gait rehabilitation of people suffering from stroke.

Methods: The study adopts a randomized, controlled, single-blinded study design. People after stroke will be eligible for participation when they are in the chronic stage of recovery ( $>6$ months after stroke), would like to improve walking performance, have a slow walking speed $(<1 \mathrm{~m} / \mathrm{s})$, can communicate in Dutch, and complete a 3-stage command. People will be excluded if they cannot walk a minimum of $10 \mathrm{~m}$ or have other additional impairments that (severely) influence gait. Participants will receive 9 gait-training sessions over a 3-week period and will be randomly allocated to an implicit or explicit group. Therapists are aware of the intervention they provide, and the assessors are blind to the intervention participants receive. Outcome will be assessed at baseline (T0), directly after the intervention (T1), and after 1 month (T2). The primary outcome parameter is walking velocity. Walking performance will be assessed with the 10-meter walking test, Dynamic Gait Index, and while performing a secondary task (dual task). Self-reported measures are the Movement Specific Reinvestment Scale, verbal protocol, Stroke and Aphasia Quality of Life Scale, and the Global Perceived Effect scale. A process evaluation will take place to identify how the therapy was perceived and identify factors that may have influenced the effectiveness of the intervention. Repeated measures analyses will be conducted to determine significant and clinical relevant differences between groups and over time.
\end{abstract}

Results: Data collection is currently ongoing and results are expected in 2019.

Conclusions: The relevance of the study as well as the advantages and disadvantages of several aspects of the chosen design are discussed, for example, the personalized approach and choice of measurements.

Trial Registration: Netherlands Trial Register NTR6272; http://www.trialregister.nl/trialreg/admin/rctview.asp?TC=6272 (Archived by WebCite http://www.webcitation.org/6ytA937m5)

Registered Report Identifier: RR1-10.2196/9595

(JMIR Res Protoc 2018;7(5):e142) doi: 10.2196/resprot.9595 


\section{KEYWORDS}

motor learning; implicit learning; explicit learning; analogy; analogy learning; gait; physiotherapy; rehabilitation; stroke; CVA

\section{Introduction}

\section{Background and Rationale}

For most people, walking is a motor skill that generally takes place without too much effort. However, for people who have suffered a stroke, walking is often suddenly impaired, which can lead to major consequences in daily life functioning. People may experience impaired walking patterns with lower walking speeds, which has been associated with lower levels of functional ambulation [1]. Gait training is one of the main components of physiotherapy within stroke rehabilitation because it is an important criterion for discharge and requirement for functioning at home [1,2]. Evidence suggests that even in later (chronic) stages after stroke, people are still able to improve motor performance [3]. Many different techniques and therapies can be used to improve walking performance [4]; the challenge for physiotherapists is to choose and deliver gait training in the most efficient and effective manner. Moreover, preferably, obtained improvements in performance are durable over a longer period and resilient under different circumstances and in dual-task situations, for example, walking and talking. Despite the availability of new training approaches such as the use of robotics [5], virtual reality, for example through exergames [6,7] or body weight support training [8], overground walking [9] still seems one of the most applied gait-training approaches in clinical practice. The current literature and clinical guidelines encourage the use of context- and task-specific treatment approaches, an example of which is overground walking $[4,10]$.

To apply overground gait training, physiotherapists are encouraged to use general motor learning principles [10]. Within the context of motor learning, a broad distinction between implicit and explicit motor learning has been described [11,12]. Explicit motor learning can be defined as learning generated by verbal knowledge of movement performance; it involves cognitive stages within the learning process and is dependent on working memory involvement [13]. The definition indicates that the learner is aware of all the underlying facts and rules of the to-be-learned motor skill during the process of learning. In practice, verbal explicit instructions are frequently used, and often these instructions encourage patients to be aware of their own body movements [14]. For example, in gait training, therapists tell patients to think about their performance, for example, "Move your hips to the left and straighten your knee before stepping" [14].

In contrast to explicit learning, implicit motor learning progresses with no or minimal increase in the verbal knowledge of movement performance (eg, facts and rules) and without awareness [13]. Learning is suggested to take place more automatically and in a less conscious manner. The learner is aware of the process of learning but cannot recall the underlying facts and rules of the motor skill. Gait training could be facilitated more implicitly, for example, when a physiotherapist would gradually constrain or change the environment, for example, when letting the person walk over different surfaces.
In this situation, verbal instructions are not needed, but the environment facilitates the motor skill (walking). An observational study demonstrated that often multiple learning strategies are being used within one training session [15]. These different learning strategies may represent an implicit-explicit continuum on which some promote more implicit and others more explicit forms of learning [11]. Looking at the current practice, therapists seem to have a preference for learning approaches that are related to explicit learning in which often high numbers of verbal explicit instructions are used [14].

Various advantages of implicit motor learning over explicit motor learning have been reported in literature [12]. Studies have demonstrated that individuals who learned motor skills implicitly perform the motor skill better under pressure, perform better in dual-task situations, and perform better over time compared with their explicit counterparts $[12,16,17]$. Although these studies have primarily been conducted within the healthy population, implicit learning may also be advantageous for the patient population $[18,19]$. For example, implicit motor learning may be of extra benefit to those with cognitive deficits [20]. Reduced cognitive function is frequently seen in people after stroke [21] and it often hampers the process of motor learning. The degree by which these cognitive functions are being evoked can be influenced by the choice of learning approaches [20]. An interesting feature of implicit motor learning is the assumption that it is less reliant on working memory resources, that is, it involves less cognitive functions, compared with explicit motor learning [19]. It is, therefore, intriguing to explore the effects of implicit motor learning within the stroke population.

Although from a theoretical perspective, the features of implicit motor learning have been described, its practical application in clinical practice seems more complex. Various learning strategies, for example, dual task, or errorless learning have been shown to promote implicit motor learning [17,22]. One learning approach that may also be placed more on the implicit side of the implicit-explicit continuum is analogy learning. In analogy learning, the learner is provided with one single analogy or metaphor that strives to combine all the relevant rules of the to-be-learned motor skill. Early studies on analogy learning took place within a sporting context, for example, to learn specific skill techniques in table tennis or basketball [23,24]. A good example in this regard was presented by Lam and colleagues [23]. They used the analogy instruction "Shoot as if you are trying to put cookies into a cookie jar on a high shelf" to teach basketball players to impart backspin on the basketball. At present, there seems an increased interest of its application within different contexts. Analogy studies have been performed with older people [25] in the context of speech therapy [26] and within neurological populations [27,28]. For example, the analogy "imagine as if you are walking over a frozen lake" has been used in gait rehabilitation to facilitate lifting and placing the foot while walking [28]. With regard to gait rehabilitation, small pilot studies have reported that analogies can be used in a feasible manner to facilitate walking performance $[27,28]$. It 
has been reported that the analogy should lead to the desired biomechanical movement and that preferably the analogy should contain a meaningful component to the participant $[28,29]$. In addition to feasibility, trends toward improved walking performance have been observed following analogy interventions, which demonstrates the potential of analogy learning in clinical practice [27,28]. However, to further establish the effectiveness of analogy learning in clinical gait rehabilitation, larger sample sizes and research designs using a control condition are required.

This study describes the design for a randomized controlled study to assess the effects of implicit motor learning compared with explicit motor learning on walking speed in people suffering from stroke. The concept of analogy learning is used to structure the gait-training sessions within the implicit condition, whereas explicit motor learning is promoted by using extensive verbal instructions and feedback. A process evaluation is embedded to investigate feasibility and fidelity of the applied interventions.

\section{Research Question}

The following research question was established to examine the effects of implicit and explicit motor learning in gait rehabilitation of people after stroke: Is a 3-week implicit motor learning walking intervention (analogies) more effective compared with a 3-week explicit motor learning walking intervention (verbal detailed instructions) delivered at home with regard to walking speed in people suffering from stroke?

\section{Methods}

\section{Study Design}

The study adopts a randomized, controlled, single-blinded study design in people suffering from stroke in the chronic stage of recovery. The study has been approved by the local ethics committee METC-Z in Heerlen, the Netherlands (NL number: NL.60338.096.16, Ethics nr: 17-T-06). After baseline measures, eligible participants will be randomized to the implicit or explicit condition (T0). Outcome assessments will take place directly after the intervention (T1) and again one month later (T2).

\section{Involvement of Client Representatives}

Throughout the design and planning of the study, 3 patient representatives were involved in every step of the decision-making process regarding the design and execution of the study. In several consensus meetings, they represented the patient's perspective, particularly with regard to the feasibility aspects of the study design. They were also involved in customizing participant information letters and promotion material for the study.

\section{Population}

The study population consists of people who had a stroke and who are living at home. People will be recruited via local private practices, rehabilitation institutes, and a local health-related newspaper. Participants will be included if they had a stroke and want to improve their gait performance. To minimize the chance that improvements occur as a result of spontaneous recovery, only participants who are in the chronic stage of recovery ( $>6$ months after stroke) will be included in the study. To prevent a ceiling effect, people with a low self-selected walking speed $(<1.0 \mathrm{~m} / \mathrm{s})$ will be invited to participate. Finally, all people should be able to communicate in Dutch and complete a 3-stage command. People are excluded if they are unable to walk a minimum distance of $10 \mathrm{~m}$; have a functional ambulation category score <3; have additional impairments not related to stroke, which influence their gait pattern, for example, severe osteoarthritis or amputation of the lower limb; have additional neurological impairments, for example, Parkinson disease that (severely) influence their walking performance.

\section{Sample Size}

The sample size calculation is based on a randomized controlled trial with equal group sizes and "walking speed" (10-meter walking test, 10MWT) as a primary outcome measure [30]. The power is set at beta $=.80$, the significance level at alpha $=.05$, and a standard deviation of $0.23 \mathrm{~m} / \mathrm{s}$ [31]. To demonstrate a significant change in walking speed, the minimal clinically important difference (MCID) is used and set at a minimum change of $0.16 \mathrm{~m} / \mathrm{s}[32,33]$. The calculations resulted in a minimum group size of 33 participants per group. Taking into account that $20 \%(7 / 33)$ of participants may be lost during (dropout) and after the intervention (loss to follow-up), this study aims to recruit 40 participants per group.

\section{Randomization, Blinding, and Treatment Allocation}

\section{Randomization Procedure}

The allocation of participants to the experimental or the control condition will occur based on a computerized randomization program. Block randomization was calculated in block sizes of fours and sixes. The randomization procedure and the randomization scheme will only be available to an independent researcher who will not be involved in the delivery of the interventions or the performance of the measurements.

\section{Blinding}

The trained assessors are blind for treatment allocation. The therapists are aware of the treatment condition as they provide the explicit or implicit motor learning condition. The participants will probably also be aware of the treatment they receive; however, they will not be specifically told. The participants will be asked at each assessment not to reveal the details of treatment they received to the blinded assessor.

\section{Training of Therapists and Treatment of Participants}

An intervention guideline is developed that outlines how the treatments (implicit and explicit conditions) should be delivered. The main aim of the intervention is to improve the quality of walking performance in people after stroke. The basic principle of the intervention guideline is based on the definitions of implicit and explicit motor learning by Kleynen et al [11]. For the purpose of this study, we strive to create the largest contrast between the conditions as possible. The main characteristics and differences in instructions, and feedback of the practice between interventions are described in Figure 1. Both conditions will always be applied to an extent that is feasible for the participant, and training will therefore always be tailored to the participant's abilities within the given boundaries of the 
condition. Similar situations will be adopted in both conditions with regard to the "organization" of the training, for example, use variation in the (analogy) instructions and practice of the motor skill.

The chosen intervention period was based on a preliminary study of Kleynen et al [28] that demonstrated that 3 weeks was a feasible period to develop and practice analogies with people after stroke. All participants will receive 9 training sessions in a 3 -week intervention period, that is, 3 training sessions per week (Figure 2). Each training session takes place at the home

Figure 1. Characteristics of the interventions. of the participants and lasts for $30 \mathrm{~min}$. Participants were asked to use the instructions in daily life (unguided therapy) and after the 3-week intervention period. To standardize the training content as much as possible, the therapists involved in the study will be trained before the start of the study. During 5 standardization training sessions, the intervention guideline will be discussed, explicated, and the therapists will be trained with the help of example cases. During the trial, therapists will attend 3 evaluation sessions to discuss the progress of the study and possible cases or difficulties they may experience during the intervention.

\begin{tabular}{|c|c|c|}
\hline & More Implicit & More Explicit \\
\hline Elements & Implicit condition & Explicit condition \\
\hline General rules & $\begin{array}{l}\text { - Minimal use of verbal instructions. } \\
\text { - For the participant, learning should } \\
\text { progress without being aware of the } \\
\text { underlying gait parameters that the } \\
\text { therapist aims to improve. }\end{array}$ & $\begin{array}{l}\text { Many verbal instructions should be } \\
\text { used, however, only to an extent that } \\
\text { is feasible for the participant. } \\
\text { - For the participant, learning should } \\
\text { progress in a conscious manner. } \\
\text { The participant should be very } \\
\text { aware of the underlying gait } \\
\text { parameters that the therapist aims to } \\
\text { improve. }\end{array}$ \\
\hline \multirow[t]{2}{*}{ Instructions } & $\begin{array}{l}\text { The instructions should only contain } \\
\text { information about the analogy. The analogy } \\
\text { can be based on: } \\
\text { - Past experiences and/or meaningful } \\
\text { situations. It should contain an image. }\end{array}$ & $\begin{array}{l}\text { Instructions should contain: } \\
\text { - Detailed instructions with reference } \\
\text { to joints, angles, and precise placing } \\
\text { of extremities/limbs. } \\
\text { - Biomechanical information. } \\
\text { - Keep internally focussed (directed } \\
\text { on body movements). } \\
\text { - Create a conscious walking } \\
\text { environment. } \\
\text { - Frequently repeat verbal } \\
\text { instructions. }\end{array}$ \\
\hline & $\begin{array}{l}\text { - No information about joints, angles, } \\
\text { position of limbs, etc. } \\
\text { - No details about spatiotemporal gait } \\
\text { parameters or biomechanics of gait. }\end{array}$ & $\begin{array}{l}\text { No information about how the } \\
\text { movement feels, no use of images, } \\
\text { no reference to familiar situations, } \\
\text { no imagery. }\end{array}$ \\
\hline Feedback & $\begin{array}{l}\text { Knowledge of results (state goal of } \\
\text { movement). } \\
\text { - Link the feedback to the analogy, eg, } \\
\text { situations, images, or emotions. } \\
\text { - Use positive feedback. }\end{array}$ & $\begin{array}{l}\text { Knowledge of performance } \\
\text { (kinematic feedback; information } \\
\text { about execution of movement). } \\
\text { - Link the instructions to, eg, feedback } \\
\text { directed at the joints, angles, or } \\
\text { placing of placing of } \\
\text { extremities/limbs. } \\
\text { - Use positive feedback. }\end{array}$ \\
\hline Examples & $\begin{array}{l}\text { - Walk as if you are walking through a } \\
\text { deep layer of snow. } \\
\text { - Walk as if you follow the footprints in the } \\
\text { sand. } \\
\text { - Try to cross a small bridge. }\end{array}$ & $\begin{array}{l}\text { - Place your heel right. } \\
\text { - } \text { - } \text { Trant your feet. } \\
\text { right/left leg. } \\
\text { - Push off with your foot. } \\
\text { - Bend your knees. } \\
\text { - Walk up-right. } \\
\text { - Lift your walking frame. }\end{array}$ \\
\hline
\end{tabular}


Figure 2. Overview of the gait training sessions.

\section{Motor learning intervention}

( 3 weeks)

\begin{tabular}{|c|c|c|c|}
\hline Sessions 1-2 & \multirow[b]{2}{*}{ Sessions 2-9 } & \multirow[b]{3}{*}{ Session 9} & \\
\hline \multirow{3}{*}{$\begin{array}{l}\text { Phase 1: Introducti } \\
\text { analyze gait, tailor } \\
\text { intervention }\end{array}$} & & & \\
\hline & \multirow{2}{*}{$\begin{array}{l}\text { Phase } 2 \text { : Training and use in } \\
\text { daily life }\end{array}$} & & (Use in daily life) \\
\hline & & $\begin{array}{l}\text { Phase 3: End } \\
\text { evaluation }\end{array}$ & ervention and \\
\hline
\end{tabular}

\section{Implicit Condition}

Within the implicit condition, the concept of analogy learning is the leading approach as it has shown to adopt characteristics of implicit motor learning [24,27,28] and provides therapists with a practical and feasible guideline to organize gait training. To choose and develop appropriate analogies, the same procedure as used by Kleynen et al is followed [28]. A booklet with examples of possible analogies will be available for the therapists and patients as a source of inspiration. Preferably, analogies are developed based on the participants' experiences and background to promote the personalized and meaningful aspects of analogy learning [28]. All analogy instructions used in the trial will be documented. In addition to analogy learning, the therapists are allowed to use elements based on the characteristics of implicit motor learning as reported under the implicit column in Figure 1.

\section{Explicit Condition}

Within the explicit condition, gait training is organized by creating a learning environment that is (more) explicit in nature. Practice will be organized based on the characteristics of explicit motor learning as reported under the explicit condition in Figure 1. The condition is similar to the first (cognitive) stage of motor learning according to Fitts and Posner [34] that is characterized by the use of many explicit instructions, explaining precisely how motor skills should be performed. This stage is verbal and cognitive of nature. Contrary to the implicit condition, the explicit condition strives to maximize the number of explicit verbal instructions. The explicit instructions that have been used in the trial will be documented by the therapist's in treatment logs.

\section{Measurements}

All measures will be assessed by independent, blinded, and trained assessors at 3 assessment points (T0, T1, and T2; Table
1) and will take place at the participant's home. The primary outcome parameter is walking speed measured in meters per second using 10MWT. First, demographics are described, and then, the primary and secondary outcome measures are reported.

\section{Demographics}

At baseline, the following demographic and prognostic information is collected (T0): age, gender, time post stroke, affected side, walking aids, educational level, cognitive level (Montreal Cognitive Assessment, MoCA) [35], static balance and fall risk (Berg Balance Scale) [36], measures of mobility and disability (Rivermead Mobility Index) [37], and ability to make movements outside the synergetic patterns (Fugl-Meyer assessment of the lower limb) [38]. To assess the propensity for conscious motor processing, the Dutch version of Movement Specific Reinvestment Scale (MSRS) is used [39,40].

\section{Walking Performance Measures}

Walking performance is measured using the 10MWT [41] and the Dynamic Gait Index (DGI) [42]. To assess the robustness of the obtained performance, walking will also be assessed over a longer period (1-month follow-up) and under secondary task loading.

\section{0-Meter Walking Test}

Gain in walking speed has been associated with a transition to a higher class of ambulation, resulting in a better function and quality of life $[41,43]$. Next to statistical significance, the MCID will be used to assess clinical relevant differences. The MCID for walking speed in people after stroke has been established at the minimal difference of $0.16 \mathrm{~m} / \mathrm{s}$ [33]. Exceeding this threshold indicates that the participants obtained a clinically meaningful improvement. 
Table 1. Overview of measurements used in this study.

\begin{tabular}{lll}
\hline Data & Time & ICF level \\
\hline Demographics & & Personal factors \\
Age, gender, time post stroke, affected or nonaffected side, walking aids, educational level & T0 & Body functions and structure \\
Montreal Cognitive Assessment & T0 & Activity level \\
$\quad$ Berg Balance Scale & T0 & Activity level \\
$\quad$ Rivermead Mobility Index & T0 Body functions and structure \\
Fugl-Meyer Assessment & T0 & Activity level \\
Walking performance & T0, T1, T2 & Activity level \\
10-meter walking test & T0, T1, T2 & Activity level \\
Dynamic Gait Index & T0, T1, T2 & Personal factors \\
Dual task & T0, T1, T2 & N/A
\end{tabular}

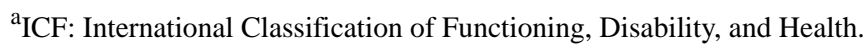

${ }^{\mathrm{b}}$ N/A: not applicable. The ICF level is not applicable for the Verbal Protocol and Global Perceived Effect Scale as these measures do not examine health or disability but evaluate the intervention.

\section{Dynamic Gait Index}

The DGI is a physical performance test that assesses the gait, balance, and fall risk and has shown to have a good reliability and validity in people after stroke [44,45]. Eight different tasks related to the balance and gait, for example, walking, turning, and stepping over objects are assessed [46]. The performance will be scored according to the modified DGI as proposed by Shumway and Cook as the extended scoring system has shown to possess good psychometric properties [42,47].

\section{Dual Task}

In this study, people will be asked to complete a tone-counting task similar to that proposed by Wilson et al [48]. In this task, people will be exposed to 4 different sounds (buzzer, ping, tone and bell ring) in a randomized order over a 30 -s time period. They will be asked to only count a specific target sound (eg, bell ring) and ignore the other 3 distracting sounds. The task will be performed twice, once as a single task and once while walking concurrently (dual task). The actual number of tones will be compared with the estimate number of tones by the participants. Error scores (actual minus estimate) will be calculated as a measure for the dual-task performance.

\section{Self-Reported Measures}

\section{Movement-Specific Reinvestment Scale Adapted for Gait}

The MSRS is a questionnaire that measures a person's inclination for conscious control. People after stroke have shown to have greater propensity to conscious processing compared with the age-matched, nondisabled population [49,50]. In this study, an adapted version of the MSRS specific to gait is used. Adapted versions of the MSRS have been used before, for example, for putting movements in golf $[51,52]$. The MSRS contains one factor related to conscious control (conscious motor processing) and one related to self-consciousness about movement (movement self-consciousness). Each factor of the MSRS comprises 5 statements, such as "I try to think about my movements when walking" (conscious motor processing) and "I am concerned about what people think about me when I am walking" (movement self-consciousness). The statements will be assessed with binary response (yes/no) [40]. The MSRS will be measured at baseline (T0) to describe the population and over time (T0, T1, and T2) to assess how much gait-related conscious processing takes place.

\section{Verbal Protocol}

To assess the amount of explicit knowledge, a verbal protocol questionnaire as used in Orrel et al will be administered after the 3-week intervention [22]. Explicit knowledge is assessed by examining the number of explicit rules that the participant uses during walking. Participants will be asked to report any "rules, methods, or techniques" that they have thought about or used and that have improved or impaired their walking performance. A rule is defined as any statement containing at least one of the following aspects: a movement or position of one limb, a movement or position of one joint, a velocity of a limb movement, an angle or direction of a joint or the spine, or the placement of the walking aid. Each statement containing a single limb, joint, or other body part will be counted as 1 rule. If a statement contains 2 (or more) different limbs, joint and body parts, or different directions or angles, they are counted separately (eg, "I tried to lift my foot and put it more forward."). Statements are excluded if they are irrelevant to walking performance or do not refer to technical aspects about walking 
(eg, "More concentration needed."). The answer to the verbal protocol will be screened by 2 independent researchers who will be blind to the experimental condition. Their agreement will be investigated using a correlation coefficient (or ICC).

\section{Stroke and Aphasia Quality of Life Scale}

The Stroke and Aphasia Quality of Life Scale ( SAQOL-39) is assessed at baseline and after the intervention to measure the health-related quality of life [53]. The questionnaire contains 39 items and is developed for people after stroke and is feasible to use for people with aphasia. The SAQOL-39 is a short version of the original SAQOL (53 items) and has shown to be an acceptable, reliable, and valid measure of the health-related quality of life [53].

\section{Global Perceived Effect}

The Global Perceived Effect scale is a reliable method to assess the participant's satisfaction and will be used to evaluate the participant's perception of the intervention [54]. The Global Perceived Effect scale will involve the following question: To which extent did your walking ability change over the last three weeks? The question will be scored on a 7-point Likert scale from "completely improved" to "completely deteriorated."

\section{Process Evaluation}

To gain an insight into the process-related factors that may have influenced the effectiveness of the 3-week analogy learning walking intervention, a process evaluation will take place along the study [55]. Data will be collected to (1) asses to what extent the therapists delivered the interventions as intended (fidelity), (2) explore the therapists' opinions and experiences about the interventions with regard to the feasibility and possible effects, and (3) explore the patients' opinions and experiences about the interventions with regard to the feasibility. Table 2 represents the data collection methods used to assess the different aspects of the process evaluation. For both groups, the provided instructions will be documented and evaluated in the therapists' logs. In addition, short questionnaires will be used to administer the therapists' and participants' opinions about the gait training after the completion of the 3-week intervention. To explore the extent to which the interventions were implicit or explicit in nature, the verbal protocol will be assessed. Evidence indicates that implicit learning is typically characterized by the less accumulation of explicit rules compared with explicit learning [24,56].

To monitor the integrity of the intervention, self-reported (subjective) and audio-recorded (objective) data will be evaluated. First, all therapists are required to self-report any deviations from the treatment protocol or other incidents during the session in a log after every session. All instructions used during therapy sessions will be recorded in the therapists' and patients' logs. Furthermore, patients can use the log to write down any possible events that might have occurred during unguided therapy. The patient log is, therefore, only used as a reminder for unguided therapy and as a communication tool between therapists and participants. Second, 10 gait trainings will be randomly selected (5 implicit and 5 explicit interventions) and audio recorded. Both self-reported and audio-recorded data will be screened to evaluate whether the intervention was delivered according to the protocol.

\section{Data Analyses}

Baseline scores of demographic and prognostic data and primary and secondary outcome measures will be used to compare the 2 groups. Only data of the participants who attended minimum of 7 or more of the therapy sessions will be considered as adherent and processed in the statistical analysis. Statistical analysis will be conducted to determine significant differences between groups and over time (baseline and postintervention performance). A repeated measure analyses will be used to compare the 2 groups (implicit and explicit) at 3 different time points (before, after, and after 1-month follow-up). Post hoc tests with correction for multiple testing will be used to explore effects over time and between groups. Subgroup analysis will be performed on cognition (MoCA score <21) [35]. An alpha level of .05 will be set for all tests. The primary outcome measure, walking speed, will also be described with reference to clinically relevant differences between groups (MCID: 0.16 $\mathrm{m} / \mathrm{s}$ ) [33]. All datasets used or analyzed during this study are available from the corresponding author on a reasonable request.

Data will be analyzed according to an intention-to-treat and per-protocol principle. In the intention-to-treat, data of the participants are analyzed according to their original treatment allocation. If self-reported (subjective) and audio-recorded (objective) data reveal that cases are not delivered as intended, then the analyses will be performed using the per-protocol principle. Within this study, protocol deviations are defined as "deviations from the protocol that occur in two or more sessions." If protocol deviations were observed, then data from this person were not included in the per-protocol analysis. Data in the process evaluation related to the therapists' and patients' opinions and experiences toward the feasibility of the intervention and perceived benefits will be analyzed by means of descriptive statistics. Free comments and clarifying examples may be quoted and used to describe personal experiences of the therapists or participants. 
Table 2. Measures for the process evaluation. A checkmark indicates with which measure the question is examined.

\begin{tabular}{|c|c|c|c|c|}
\hline \multirow[t]{2}{*}{ Questions } & \multicolumn{4}{|l|}{ Measures } \\
\hline & Therapist Log & Audio recordings & Therapist questionnaire & Patient questionnaire \\
\hline $\begin{array}{l}\text { To what extent did the therapists deliver the interventions } \\
\text { as intended (fidelity)? }\end{array}$ & $\checkmark$ & $\checkmark$ & $\checkmark$ & \\
\hline $\begin{array}{l}\text { How did the therapist's experience delivering the interven- } \\
\text { tions with regard to the feasibility and possible effects? }\end{array}$ & & & $\checkmark$ & \\
\hline $\begin{array}{l}\text { How did the patients' experience the intervention with regard } \\
\text { to the feasibility? }\end{array}$ & & & & $\checkmark$ \\
\hline
\end{tabular}

\section{Results}

The entire project was funded in September 2015. Patient enrolment began in March 2017 and is expected to continue until July 2018. Following completion of data collection, data cleaning and analyses will take place. The first study results are expected to be submitted for publication in 2019 .

\section{Discussion}

In this paper, we described the methodology of a randomized controlled single-blinded study that evaluates the potential effects of implicit motor learning compared with explicit motor learning in the gait rehabilitation of people suffering from stroke. The relevance of the study and the advantages and disadvantages of several aspects of the chosen design are discussed.

\section{From Laboratory Setting to Clinical Practice}

Although motor learning research is growing exponentially, most published studies have been performed in laboratory settings. However, it is important to understand the application of motor learning within clinically relevant environments and the influence of interventions on the completion of everyday functional tasks $[18,57]$. A recent systematic review on implicit motor learning in people after stroke pointed out that studies performed within clinical settings are limited [18]. Of the 20 included studies, only 1 study involved a clinically relevant task [22]. To improve the generalizability of research findings toward clinical practice and to the broader population, various choices on different aspects in the research design had to be made. First, with regard to the task and environment, this study involves gait training that takes place at the homes of the participants. Gait is a functional daily life motor skill, and it is advised to organize practice in a context-specific environment [4,58]. Therefore, compared with the current state of evidence, this study adds insights into the effects of motor learning in a clinically relevant environment and for a clinically relevant task.

Second, in this study, the motor learning conditions are tailored to the individual participants. Most implicit motor learning studies use strict research protocols in which each participant usually receives the exact same instructions (eg, the whole experimental group receives the same right-angled triangle analogy to learn topspin forehand in table tennis), whereas the explicit learning is promoted by using the same set of verbal instructions [24]. A one-size-fits-all approach may not be ideal for the clinical population as they generally demonstrate a large variety in degree and types of impairments. The use of personalized analogies allows the physiotherapists to respond to the individual walking impairments and emphasize on the meaningful component of analogy learning. With a personalized approach (more comparable with the real-life practice), the instructions may be less standardized. Therefore, steps were undertaken to ensure the quality of the interventions. Before the study, all therapists were familiarized and trained with the implicit and explicit conditions. Measures (therapists' logs and audio recordings of the interventions) were selected to evaluate whether the trainings were delivered as intended, and data will be analyzed as per the intention-to-treat and per-protocol (see the Data Analyses section).

\section{Methodological Aspects}

Within this study, extra attention was given to the following 3 methodological aspects in designing the intervention: contrast, content of the interventions, and target population. First of all, it is important to address that in many rehabilitation studies, the contrast between the intervention and control group (generally usual care only) turns out to be too modest, which therefore results in neutral study results [59]. The underlying reason may be that rehabilitation interventions are often too complex to control for influences of other interventions and that the control intervention group (therapy as usual) is often poorly described. We tried to overcome these problems by including participants in the chronic phase of recovery, who do not receive additional interventions directed at the improvement of gait. Furthermore, we strive to ensure the contrast of the interventions by using a guideline, written protocol. In this protocol, the delivery of both conditions is clearly defined. It transparently describes how the 2 interventions differentiate from each other. To ensure the integrity of the intervention, the logs and audio-recorded therapy sessions will be conducted and evaluated.

Another decision we would like to address is the choice for the interventions. In clinical practice, often mixtures of implicit and explicit forms of learning are used or therapists switch between different forms of learning $[15,60]$. However, it remains unclear whether mixing implicit and explicit learning or switching between the forms is effective and/or necessary. The current design is necessary to evaluate the effects of implicit versus explicit learning in clinical practice, and so we believe that it is (ethically) legitimate to compare 2 distinct interventions and not use "therapy as usual" as a control intervention. The interventions in this study will be delivered in a personalized manner regarding overall components of the interventions, for example, the sort of gait impairments, amount of repetitions, and use of personalized analogies. However, the content of the 
interventions applied is clearly outlined in the guidelines, and therapists are required to strictly follow these.

Furthermore, within the target population, people experiencing cognitive impairments or communicative restrictions are often excluded. In this study, we strive to include a sample that reflects the broad range of impairments reported after stroke. In earlier studies, it has been shown that motor learning interventions might be effective, also for people with cognitive and/or communicative impairments [27,28].

\section{Implicit Motor Learning}

Scientific evidence describes that implicit learning is typically characterized by robust dual-task performance, durable performance over time, and less accumulation of explicit rules [24,56]. The dual-task measure and the 1-month follow-up session were specifically chosen with respect to these implicit characteristics. First, walking while concurrently carrying out a secondary (tone counting) task [48] places high information-processing demands on the learners. In contrast to explicit learners, implicit learners showed that performance was not disrupted in dual-task conditions, which indicates that they may be able to free up attentional resources to perform the secondary task [24]. We therefore predict that the implicit condition will remain stable under secondary task burden. For this reason, implicit learning may be particularly beneficial for those who experience cognitive impairments that are commonly seen in people after stroke.
Then, a recent study by Tse et al [25] found performance improvements after a 2-day separation; however, they recommended to include a longer separation to test skill consolidation. This study will therefore evaluate performance after the 1-month follow-up period. It is hypothesized that performance improvements in the implicit group will remain robust over a longer period (1-month follow-up). To make statements about long-term effects within rehabilitation, it may be desirable for future studies to include even longer follow-up periods ( $>3$ months). Finally, a verbal protocol will be assessed because it is hypothesized that participants within the implicit learning group will report fewer explicit rules than the control group, which would be in line with the findings of earlier studies $[23,24]$. Even though the starting point of the explicit intervention is to use many verbal explicit instructions and provide more details on the motor performance, the exact number of explicit rules will be tailored to the ability of the patient to process these rules. Therefore, in practice, some patients might receive higher numbers of explicit rules than others. Still, we hypothesize that the explicit group will require more explicit rules compared with the implicit group.

\section{Conclusion and Implementation}

With the description of the study design, we hope to contribute to the discussion on how a tailored but standardized form of implicit motor learning could be applied in clinical practice. The relevance of the study and the advantages and disadvantages of several aspects of the chosen design are discussed (eg, personalized approach, sample selection).

\section{Acknowledgments}

The authors would like to thank all (consortium) partners of the research project "Power of Implicit Motor Learning 2.0" for their participation in research meetings and thoughts along the planning and design of the study.

The authors would like to thank the therapists and researchers Jos Halfens, Jos Kurvers, Kyra Theunissen, Monique Rothbauer, Inge de Lang, and Ritch te Kampen for their expertise and thoughts in designing the therapist's guidance and measurement protocols. The authors would also like to thank their client representatives Nathalie Sieben, Else de Bont, and Anja Minheere for their valuable perspectives and thoughts about the trial along the set-up of the study. The authors would like to thank Kirsty Brock for helping improve the language of the manuscript. This study is financially supported by Stichting Alliantie Innovatie (Innovation Alliance Foundation), RAAK-international (registration number: 2011-3-33int).

\section{Authors' Contributions}

LJ, MK, AB, and SB conceived and designed the study. LJ, MK, and SB wrote the first version of the manuscript. LJ, MK, KM, $\mathrm{AB}$, and $\mathrm{SB}$ critically revised the manuscript for the relevant intellectual content. All authors read and approved the final version for submission.

\section{Conflicts of Interest}

None declared.

\section{Multimedia Appendix 1}

Peer review report by Dutch sponsor, Stichting Innovatie Alliantie (Innovation Alliance Foundation).

[PDF File (Adobe PDF File), 161KB-Multimedia Appendix 1]

\section{References}

1. Perry J, Garrett M, Gronley JK, Mulroy SJ. Classification of walking handicap in the stroke population. Stroke 1995 Jun;26(6):982-989 [FREE Full text] [Medline: 7762050] 
2. Dobkin BH. Clinical practice. Rehabilitation after stroke. N Engl J Med 2005 Apr 21;352(16):1677-1684 [FREE Full text] [doi: 10.1056/NEJMcp043511] [Medline: 15843670]

3. Krakauer JW. Motor learning: its relevance to stroke recovery and neurorehabilitation. Curr Opin Neurol 2006 Feb;19(1):84-90. [Medline: 16415682 ]

4. Belda-Lois J, Mena-del Horno S, Bermejo-Bosch I, Moreno JC, Pons JL, Farina D, et al. Rehabilitation of gait after stroke: a review towards a top-down approach. J Neuroeng Rehabil 2011 Dec 13;8:66 [FREE Full text] [doi: 10.1186/1743-0003-8-66] [Medline: 22165907]

5. Pennycott A, Wyss D, Vallery H, Klamroth-Marganska V, Riener R. Towards more effective robotic gait training for stroke rehabilitation: a review. J Neuroeng Rehabil 2012 Sep 07;9:65 [FREE Full text] [doi: 10.1186/1743-0003-9-65] [Medline: 22953989]

6. Moreira MC, de Amorim Lima AM, Ferraz KM, Benedetti Rodrigues MA. Use of virtual reality in gait recovery among post stroke patients--a systematic literature review. Disabil Rehabil Assist Technol 2013 Sep;8(5):357-362. [doi: 10.3109/17483107.2012.749428] [Medline: 23614694]

7. Molina KI, Ricci NA, de Moraes SA, Perracini MR. Virtual reality using games for improving physical functioning in older adults: a systematic review. J Neuroeng Rehabil 2014;11:156 [FREE Full text] [doi: 10.1186/1743-0003-11-156] [Medline: 25399408]

8. Mehrholz J, Pohl M, Elsner B. Treadmill training and body weight support for walking after stroke. Cochrane Database Syst Rev 2014 Jan 23(1):CD002840. [doi: 10.1002/14651858.CD002840.pub3] [Medline: 24458944]

9. States RA, Salem Y, Pappas E. Overground gait training for individuals with chronic stroke: a Cochrane systematic review. J Neurol Phys Ther 2009 Dec;33(4):179-186. [doi: 10.1097/NPT.0b013e3181c29a8c] [Medline: 20208461]

10. Pollock A, Baer G, Campbell P, Choo PL, Forster A, Morris J, et al. Physical rehabilitation approaches for the recovery of function and mobility after stroke: major update. Stroke 2014;45:-. [doi: 10.1161/STROKEAHA.114.006275]

11. Kleynen M, Braun SM, Rasquin SMC, Bleijlevens MHC, Lexis MAS, Halfens J, et al. Multidisciplinary views on applying explicit and implicit motor learning in practice: an international survey. PLoS One 2015;10(8):e0135522 [FREE Full text] [doi: 10.1371/journal.pone.0135522] [Medline: 26296203]

12. Hodges N, Williams AM, editors. Advances in implicit motor learning. In: Skill Acquisition in Sport: Research, Theory and Practice. Abingdon, United Kingdom: Routledge; 2012:59-75.

13. Kleynen M, Braun SM, Bleijlevens MH, Lexis MA, Rasquin SM, Halfens J, et al. Using a Delphi technique to seek consensus regarding definitions, descriptions and classification of terms related to implicit and explicit forms of motor learning. PLoS One 2014;9(6):e100227 [FREE Full text] [doi: 10.1371/journal.pone.0100227] [Medline: 24968228]

14. Johnson L, Burridge JH, Demain SH. Internal and external focus of attention during gait re-education: an observational study of physical therapist practice in stroke rehabilitation. Phys Ther 2013 Jul;93(7):957-966. [doi: 10.2522/ptj.20120300] [Medline: $\underline{23559523}$ ]

15. Kleynen M, Moser A, Haarsma FA, Beurskens AJ, Braun SM. Physiotherapists use a great variety of motor learning options in neurological rehabilitation, from which they choose through an iterative process: a retrospective think-aloud study. Disabil Rehabil 2017;39(17):1729-1737. [doi: 10.1080/09638288.2016.1207111] [Medline: 27440392]

16. Dienes Z, Berry D. Implicit learning: below the subjective threshold. Psychon Bull Rev 1997;4(1):3-23. [doi: 10.3758/BF03210769]

17. Masters RSW. Knowledge, knerves and know-how: The role of explicit versus implicit knowledge in the breakdown of a complex motor skill under pressure. Brit J Psychol 1992;83(3):343-358. [doi: 10.1111/j.2044-8295.1992.tb02446.x]

18. Kal E, Winters M, van der Kamp J, Houdijk H, Groet E, van Bennekom C, et al. Is implicit motor learning preserved after stroke? A systematic review with meta-analysis. PLoS One 2016;11(12):e0166376 [FREE Full text] [doi: 10.1371/journal.pone.0166376] [Medline: 27992442]

19. Steenbergen B, van der Kamp J, Verneau M, Jongbloed-Pereboom M, Masters RS. Implicit and explicit learning: applications from basic research to sports for individuals with impaired movement dynamics. Disabil Rehabil 2010;32(18):1509-1516. [doi: 10.3109/09638288.2010.497035] [Medline: 20575752]

20. Lee TD, Swinnen SP, Serrien DJ. Cognitive effort and motor learning. Quest 1994;46(3):328-344. [doi: 10.1080/00336297.1994.10484130]

21. Hochstenbach J, Mulder T, van Limbeek J, Donders R, Schoonderwaldt H. Cognitive decline following stroke: a comprehensive study of cognitive decline following stroke. J Clin Exp Neuropsychol 1998 Aug;20(4):503-517. [doi: 10.1076/jcen.20.4.503.1471] [Medline: 9892054]

22. Orrell AJ, Eves FF, Masters RS. Motor learning of a dynamic balancing task after stroke: implicit implications for stroke rehabilitation. Phys Ther 2006 Mar;86(3):369-380. [Medline: 16506873]

23. Lam WK, Maxwell JP, Masters RS. Analogy versus explicit learning of a modified basketball shooting task: performance and kinematic outcomes. J Sports Sci 2009 Jan 15;27(2):179-191. [doi: 10.1080/02640410802448764] [Medline: 19153868]

24. Liao CM, Masters RS. Analogy learning: a means to implicit motor learning. J Sports Sci 2001 May;19(5):307-319. [doi: 10.1080/02640410152006081] [Medline: $\underline{11354610]}$

25. Tse ACY, Wong TWL, Masters RSW. Examining motor learning in older adults using analogy instruction. Psychol Sport Exerc 2017;28:78-84. [doi: 10.1016/j.psychsport.2016.10.005] 
26. Tse CY, Wong A, Whitehill T, Ma E, Masters R. Examining the cognitive demands of analogy instructions compared to explicit instructions. Int J Speech Lang Pathol 2016 Oct;18(5):465-472. [doi: 10.3109/17549507.2015.1112834] [Medline: 27063681]

27. Jie L, Goodwin V, Kleynen M, Braun S, Nunns M, Wilson M. Analogy learning in Parkinson's disease: a proof-of-concept study. Int J Ther Rehabil 2016;23(3):-. [doi: 10.12968/ijtr.2016.23.3.123]

28. Kleynen M, Wilson MR, Jie LJ, te Lintel Hekkert F, Goodwin VA, Braun SM. Exploring the utility of analogies in motor learning after stroke: a feasibility study. Int J Rehabil Res 2014 Sep;37(3):277-280. [doi: 10.1097/MRR.0000000000000058] [Medline: 24681769]

29. Poolton J, Masters RS, Maxwell JP. The development of a culturally appropriate analogy for implicit motor learning in a Chinese population. Sport Psychol 2007;21(4):375-382. [doi: 10.1123/tsp.21.4.375]

30. Rosner B. Hypothesis testing: two-sample inference / estimation of sample size and power for comparing two means. In: Fundamentals of biostatistics, 7th edition. Boston, Massachusetts, United States: Cengage Learning; 2010.

31. Combs-Miller SA, Kalpathi Parameswaran A, Colburn D, Ertel T, Harmeyer A, Tucker L, et al. Body weight-supported treadmill training vs. overground walking training for persons with chronic stroke: a pilot randomized controlled trial. Clin Rehabil 2014 Sep;28(9):873-884. [doi: 10.1177/0269215514520773] [Medline: 24519922]

32. Brach JS, Perera S, Studenski S, Katz M, Hall C, Verghese J. Meaningful change in measures of gait variability in older adults. Gait Posture 2010 Feb;31(2):175-179 [FREE Full text] [doi: 10.1016/j.gaitpost.2009.10.002] [Medline: 19889543]

33. Tilson JK, Sullivan KJ, Cen SY, Rose DK, Koradia CH, Azen SP, Locomotor Experience Applied Post Stroke (LEAPS) Investigative Team. Meaningful gait speed improvement during the first 60 days poststroke: minimal clinically important difference. Phys Ther 2010 Feb;90(2):196-208 [FREE Full text] [doi: 10.2522/ptj.20090079] [Medline: 20022995]

34. Fitts PM, Posner MI. Learning and skilled performance in human performance. Belmont, CA: Brooks/Cole Publishing; 1967.

35. Nasreddine ZS, Phillips NA, Bédirian V, Charbonneau S, Whitehead V, Collin I, et al. The Montreal Cognitive Assessment, MoCA: a brief screening tool for mild cognitive impairment. J Am Geriatr Soc 2005 Apr;53(4):695-699. [doi: 10.1111/j.1532-5415.2005.53221.x] [Medline: 15817019$]$

36. Berg K, Wood-Dauphine S, Williams J, Gayton D. Measuring balance in the elderly: preliminary development of an instrument. Physiother Can 1989;41(6):304-311. [doi: 10.3138/ptc.41.6.304]

37. Collen FM, Wade DT, Robb GF, Bradshaw CM. The Rivermead Mobility Index: a further development of the Rivermead Motor Assessment. Int Disabil Stud 1991;13(2):50-54. [Medline: 1836787]

38. Fugl-Meyer AR, Jääskö L, Leyman I, Olsson S, Steglind S. The post-stroke hemiplegic patient. 1. a method for evaluation of physical performance. Scand J Rehabil Med 1975;7(1):13-31. [Medline: 1135616]

39. Masters RSW, Polman RCJ, Hammond NV. 'Reinvestment': a dimension of personality implicated in skill breakdown under pressure. Pers Indiv Differ 1993;14(5):655-666. [doi: 10.1016/0191-8869(93)90113-H]

40. Kleynen M, Braun SM, Beurskens AJ, Verbunt JA, de Bie RA, Masters RS. Investigating the Dutch Movement-Specific Reinvestment Scale in people with stroke. Clin Rehabil 2013 Feb;27(2):160-165. [doi: 10.1177/0269215512448381] [Medline: 22801473]

41. Collen FM, Wade DT, Bradshaw CM. Mobility after stroke: reliability of measures of impairment and disability. Int Disabil Stud 1990;12(1):6-9. [Medline: 2211468]

42. Shumway-Cook A, Taylor CS, Matsuda PN, Studer MT, Whetten BK. Expanding the scoring system for the Dynamic Gait Index. Phys Ther 2013 Nov;93(11):1493-1506. [doi: 10.2522/ptj.20130035] [Medline: 23813090]

43. Schmid A, Duncan PW, Studenski S, Lai SM, Richards L, Perera S, et al. Improvements in speed-based gait classifications are meaningful. Stroke 2007 Jul;38(7):2096-2100 [FREE Full text] [doi: 10.1161/STROKEAHA.106.475921] [Medline: 17510461]

44. Jonsdottir J, Cattaneo D. Reliability and validity of the dynamic gait index in persons with chronic stroke. Arch Phys Med Rehabil 2007 Nov;88(11):1410-1415. [doi: 10.1016/j.apmr.2007.08.109] [Medline: 17964880]

45. Shumway-Cook A, Woollacott M. Motor Control: Theory and Practical Applications. Philadelphia: Lippincott Williams \& Wilkins; 1995.

46. Shumway-Cook A, Baldwin M, Polissar NL, Gruber W. Predicting the probability for falls in community-dwelling older adults. Phys Ther 1997 Aug;77(8):812-819. [Medline: 9256869]

47. Matsuda PN, Taylor CS, Shumway-Cook A. Evidence for the validity of the modified dynamic gait index across diagnostic groups. Phys Ther 2014 Jul;94(7):996-1004. [doi: 10.2522/ptj.20130294] [Medline: 24557650]

48. Wilson MR, Vine SJ, Bright E, Masters RS, Defriend D, McGrath JS. Gaze training enhances laparoscopic technical skill acquisition and multi-tasking performance: a randomized, controlled study. Surg Endosc 2011 Dec;25(12):3731-3739 [FREE Full text] [doi: 10.1007/s00464-011-1802-2] [Medline: 21671125]

49. Orrell AJ, Masters RS, Eves FF. Reinvestment and movement disruption following stroke. Neurorehabil Neural Repair 2009 Feb;23(2):177-183. [doi: 10.1177/1545968308317752] [Medline: 18987385]

50. Denneman RPM, Kal EC, Houdijk H, Kamp JV. Over-focused? The relation between patients' inclination for conscious control and single- and dual-task motor performance after stroke. Gait Posture 2018 Mar 5;62:206-213. [doi:

10.1016/j.gaitpost.2018.03.008] [Medline: 29571088] 
51. Cooke A, Kavussanu M, McIntyre D, Boardley ID, Ring C. Effects of competitive pressure on expert performance: underlying psychological, physiological, and kinematic mechanisms. Psychophysiology 2011 Aug;48(8):1146-1156. [doi: 10.1111/j.1469-8986.2011.01175.x] [Medline: 21265862]

52. Vine S, Moore L, Cooke A, Ring C, Wilson M. Quiet eye training: a means to implicit motor learning. Int J Sport Psychol 2013;44(4):367-386.

53. Hilari K, Byng S, Lamping DL, Smith SC. Stroke and Aphasia Quality of Life Scale-39 (SAQOL-39): evaluation of acceptability, reliability, and validity. Stroke 2003 Aug;34(8):1944-1950 [FREE Full text] [doi: 10.1161/01.STR.0000081987.46660.ED] [Medline: 12855827]

54. Hudak PL, Wright JG. The characteristics of patient satisfaction measures. Spine (Phila Pa 1976) 2000 Dec 15;25(24):3167-3177. [Medline: 11124733]

55. Saunders RP, Evans MH, Joshi P. Developing a process-evaluation plan for assessing health promotion program implementation: a how-to guide. Health Promot Pract 2005 Apr;6(2):134-147. [doi: 10.1177/1524839904273387] [Medline: 15855283]

56. Berry DC, Dienes Z. Implicit learning: Theoretical and empirical issues. United Kingdom: Psychology Press; 1993.

57. Fisher BE, Morton SM, Lang CE. From motor learning to physical therapy and back again: the state of the art and science of motor learning rehabilitation research. J Neurol Phys Ther 2014 Jul;38(3):149-150 [FREE Full text] [doi:

10.1097/NPT.0000000000000043] [Medline: 24806437]

58. KNGF Clinical Practice Guideline for Physical Therapy in patients with stroke. Amersfoort: Royal Dutch Society for Physical Therapy (Koninklijk Nederlands Genootschap voor Fysiotherapie, KNGF); 2014.

59. Winstein CJ, Wolf SL, Dromerick AW, Lane CJ, Nelsen MA, Lewthwaite R, Interdisciplinary Comprehensive Arm Rehabilitation Evaluation (ICARE) Investigative Team. Effect of a task-oriented rehabilitation program on upper extremity recovery following motor stroke: the ICARE randomized clinical trial. J Am Med Assoc 2016 Feb 9;315(6):571-581 [FREE Full text] [doi: 10.1001/jama.2016.0276] [Medline: 26864411]

60. Kal E, van den Brink H, Houdijk H, van der Kamp J, Goossens PH, van Bennekom C, et al. How physical therapists instruct patients with stroke: an observational study on attentional focus during gait rehabilitation after stroke. Disabil Rehabil 2017 Feb 24:1-12. [doi: 10.1080/09638288.2017.1290697] [Medline: 28637152]
Abbreviations
10MWT: 10-meter walking test
DGI: Dynamic Gait Index
MCID: minimal clinically important difference
MSRS: Movement Specific Reinvestment Scale
SAQOL-39-NL: Stroke and Aphasia Quality Of Life

Edited by G Eysenbach; submitted 07.12.17; peer-reviewed by A Kerr, R Islam, D Taylor; comments to author 17.03.18; revised
version received 30.03.18; accepted 31.03.18; published 24.05.18
Please cite as:
Jie LJ, Kleynen M, Meijer K, Beurskens A, Braun S
The Effects of Implicit and Explicit Motor Learning in Gait Rehabilitation of People After Stroke: Protocol for a Randomized Controlled
Trial
JMIR Res Protoc 2018;7(5):e142
URL: $\underline{\text { http://www.researchprotocols.org/2018/5/e142/ }}$
doi: $\underline{10.2196 / \text { resprot.9595 }}$
PMID: $\underline{29793902}$

CLi-Juan Jie, Melanie Kleynen, Kenneth Meijer, Anna Beurskens, Susy Braun. Originally published in JMIR Research Protocols (http://www.researchprotocols.org), 24.05.2018. This is an open-access article distributed under the terms of the Creative Commons Attribution License (https://creativecommons.org/licenses/by/4.0/), which permits unrestricted use, distribution, and reproduction in any medium, provided the original work, first published in JMIR Research Protocols, is properly cited. The complete bibliographic information, a link to the original publication on http://www.researchprotocols.org, as well as this copyright and license information must be included. 